DOI: https://doi.org/10.34069/RA/2020.6.01

Volumen 3, Número 6/julio-diciembre 2020

\title{
Caracterización etnobotánica de la Lawsonia inermis L. en el Distrito José Martí Norte, Santiago de Cuba.
}

\section{Ethnobotanical characterization of Lawsonia inermis L. in the José Martí Norte District, Santiago de Cuba.}

\author{
Recibido: 27 de julio de $2020 \quad$ Aceptado: 25 de octubre de 2020
}

\author{
Autores: \\ Imilci Urdaneta Laffita ${ }^{1}$ \\ Lourdes Padró Rodríguez ${ }^{1}$ \\ Enieyis Tur Naranjo ${ }^{1}$ \\ Yonal Luis Izaguirre Hernández ${ }^{1}$
}

\section{Resumen}

Se realizó un estudio etnobotánico de la especie Lawsonia inermis L. en el Consejo Popular "Distrito José Martí Norte", municipio Santiago de Cuba, con el objetivo de caracterizar el uso de la especie, auxiliándonos de la metodología establecida para estudios etnobotánicos, empleando como instrumento una entrevista apoyada en la encuesta establecida en el programa de investigación aplicada a la medicina popular del Caribe (TRAMIL). Se calcularon los indicadores etnobotánicos cuantitativos: índice de valor de uso (IVU) e índice de nivel de uso significativo (NUS). Fueron entrevistadas 500 personas. El sexo predominante fue el femenino (64\%); el grupo etario comprendido entre 31-40 años resultó ser el más entrevistado; el nivel de escolaridad bachiller $(36,8 \%)$ y la categoría de trabajadores $(68,6 \%)$ fueron los más representativos dentro de sus respectivas categorías. Tienen conocimiento de la especie 426 personas $(85,2 \%)$ y de estas la utilizan $327(76,7 \%)$. Se informaron siete usos, destacándose el de calmar los nervios con 312 citaciones, representado un 95,4\%. Las partes aéreas de la planta fueron las más utilizadas. El $91 \%$ de los entrevistados emplean el método de infusión para la obtención del producto medicinal utilizando la planta en estado fresco mayormente. La vía oral resultó la forma de administración más reportada (98\%). Los valores de los índices etnobotánicos cuantitativos correspondieron a un IVU igual a 0,65 y un NUS de $65 \%$ para el Consejo Popular, siendo la localidad Micro 9 la que evidenció un mayor conocimiento de uso de la especie.

Palabras claves: estudio etnobotánico, Lawsonia inermis L, plantas medicinales, usos tradicionales.

\begin{abstract}
An ethnobotanical study of the species Lawsonia inermis L. was carried out in the Popular Council "District Jose Martí Norte", municipality of Santiago de Cuba, with the objective of

\footnotetext{
${ }^{1}$ Licenciada en Ciencias Farmacéuticas. Profesor Asistente. Departamento de Farmacia, Facultad de Ciencias Naturales y Exactas, Universidad de Oriente, Santiago de Cuba, Cuba. ORCID: https://orcid.org/0000-0002-5047-5771

${ }^{1}$ Licenciada en Ciencias Farmacéuticas. Profesor Auxiliar. Departamento de Farmacia, Facultad de Ciencias Naturales y Exactas, Universidad de Oriente, Santiago de Cuba, Cuba. ORCID: https://orcid.org/0000-0002-1256-8547

${ }^{1}$ Licenciada en Biología. Profesor Auxiliar. Departamento de Farmacia, Facultad de Ciencias Naturales y Exactas, Universidad de Oriente, Santiago de Cuba, Cuba. ORCID: https://orcid.org/0000-0003-4626-1962

${ }^{1}$ Licenciado en Ciencias Farmaceúticas. Departamento de Farmacia, Facultad de Ciencias Naturales y Exactas, Universidad de Oriente, Santiago de Cuba, Cuba. ORCID: https://orcid.org/0000-0002-8131-2748
} 
characterizing the use of the species, aiding in the established methodology for ethnobotanical studies, using as Instrument an interview based on a survey that established the research program applied to the Caribbean popular medicine (TRAMIL). Quantitative ethnobotanical indicators were calculated: value of use index (IVU) and index of significant use level (NUS). 500 people were interviewed. The sex with the highest prevalence was female (64\%); the most interviewed age group corresponded to between 31-40 years old; the senior high school level $(36,8 \%)$ and the worker cathegory $(68,6 \%)$ were the most represented within their respective categories. 426 people $(85,2 \%)$ are aware of the specie and $327(76,7 \%)$ of them use it. Seven uses were reported, the most reported being to calm the nerves with $312(95,4 \%)$ citations. The aerial parts of the plant were the most used. $91 \%$ of the interviewed used the infusion method to obtain the medicinal product using the plant in the fresh state. The oral route resulted the most reported (98\%) form of administration. The values of the quantitative ethnobotanical indexes were of a value of IVU of 0.65 and NUS of $65 \%$ for the Popular Council, being Micro 9 locality that showed a greater knowledge of use of the specie.

Keywords: ethnobotanical studies, Lawsonia inermis L, medicinal plants, tradicionals uses.

\section{Introducción}

Desde tiempos muy antiguos el hombre descubrió que las plantas no sólo satisfacen sus necesidades de subsistencia, sino que también, curan sus enfermedades (Ochoa et al., 1995) por lo que hoy en día son utilizadas como medicamentos herbolarios para diferentes padecimientos y se comercializan por todo el mundo (Illnait, 2007).

La Organización Mundial de la Salud (OMS) (OMS, 2002), señala que el 80\% de la población mundial utiliza las plantas como principal remedio medicinal debido al conocimiento empírico, transmitido de generación en generación, que ha llevado a su utilización en las primeras necesidades de la atención médica (Pérez et al, 2011), pero muy pocas especies y productos se han estudiado con fines médicos y un número menor cuenta con estudios realizados sobre su seguridad, eficacia y calidad.

Cuba, posee una valiosa tradición en la utilización de las plantas medicinales y cuenta con una flora muy diversa que permite su explotación racional. Sin embargo, existen muchas especies vegetales que no están avaladas científicamente, y que son usadas por la población sin conocer los riesgos que estas pueden ocasionar, cobrando gran importancia el estudio de las mismas (Macías et al., 2011).

Tal es el caso de la Lawsonia inermis L., perteneciente a la familia Lythraceae y conocida por el nombre vulgar resedá, aunque en el mundo también se le conoce por otros sinónimos como henna, alheña y mendi (Gagandeep et al., 2010; Jain, 2010; Santosh et al., 2013). Esta especie es muy utilizada en la medicina tradicional para el tratamiento de varias dolencias como artritis reumatoidea, úlceras, dolor de cabeza, fiebre, diarrea, cálculos renales, epilepsia, histeria, insomnio, afecciones de la piel, enfermedades cardíacas y hepáticas (Gagandeep et al., 2010; Kumar et al., 2016; Sharma et al., 2016). Sin embargo, posee escasos reportes de estudios científicos en el país que justifiquen el amplio uso que le dan los pobladores, principalmente en el tratamiento de las afecciones nerviosas como la ansiedad y el insomnio, a excepción del estudio realizado por Hernández J. en el año 2004 donde se informa el uso etnomedicinal de la Lawsonia inermis L. en la región más oriental de Cuba (provincias Santiago de Cuba y Guantánamo) para tratar estas dolencias dado a su efecto sedante, lo que nos motiva a realizar investigaciones científicas encaminadas a la búsqueda de conocimientos que nos permita evaluar y validar científicamente dicha especie. 


\section{AMAZONANAS}

Basados en que los estudios etnobotánicos realizados en Cuba (Pimentel, 2002; Beyra et al, 2004; Pérez et al, 2009; Vargas et al, 2011), han estado encaminados a la búsqueda de información necesaria para la investigación farmacológica de aquellas especies vegetales con un rico acervo cultural y tradicional, a fin de identificar nuevos agentes terapéuticos con menor riesgo de toxicidad y aparición de efectos secundarios, a partir del uso popular que le confiere la población, los cuales resultan muy diversos y variables (Pérez et al., 2009) y tomando en consideración la no existencia de estudios etnobotánicos para la especie Lawsonia inermis L. en Cuba, y del hecho que el criterio etnobotánico es en la actualidad el más aceptado para el estudio de especies vegetales potenciales que puedan conducir al desarrollo de nuevos medicamentos, y que constituyen el punto de partida para investigar acerca del uso de dicha especie a partir del conocimiento empírico que se tiene de la misma, es que se hace necesario realizar esta investigación la cual está encaminada a conocer el estado actual del uso tradicional de dicha especie, pudiendo sentar las bases para futuras investigaciones dirigidas a la búsqueda de principios activos y nuevas alternativas terapéuticas.

Teniendo en cuenta los antecedentes citados nos proponemos como objetivo de este trabajo: Caracterizar el uso de la especie Lawsonia inermis L. en el Consejo Popular "Distrito José Martí Norte", del municipio Santiago de Cuba.

\section{Marco teórico}

Etnobotánica. Es el área del conocimiento que estudia las relaciones e interrelaciones entre las plantas y los humanos. Tiene como principal objetivo la búsqueda del conocimiento sobre las plantas y rescate de sus utilidades en la cultura popular tradicional (Pardo, Gómez, 2003).

Informantes. Personas que comparten el saber etnobotánico colectivo cuyos testimonios se recopilan a través de registros audiovisuales, cuadernos y otros instrumentos. La metodología de obtención de datos son las entrevistas y encuestas, que pueden ser abierta o estructurada, individual o colectiva, etcétera (Rodríguez et al., 2019).

Encuestas etnobotánicas. Consisten en la recolección de los usos dados a las plantas medicinales por los pobladores (Martin, 1995). El objetivo es conocer las plantas y sus partes utilizadas, el modo de preparación y dosis de tratamiento, y las enfermedades para las que le sirven al ser humano (Blanché et al., 1996).

\section{Metodología}

Se realizó un estudio etnobotánico con un enfoque cualitativo y cuantitativo en el Consejo Popular "Distrito José Martí Norte" del municipio Santiago de Cuba, provincia Santiago de Cuba, en el período comprendido entre los meses de enero y mayo del 2018, con el objetivo de caracterizar desde el punto de vista etnobotánico la especie vegetal Lawsonia inermis L.

\section{Descripción del área de estudio}

La región geográfica objeto de estudio fue el Consejo Popular "Distrito José Martí Norte", corresponde al Distrito poblacional \#1 "José Martí" del municipio Santiago de Cuba, perteneciente a la provincia del mismo nombre. Comprende las localidades de los Micros 9 y 10, La Risueña, Los Cocos, Altos de Quintero y Bajo Rancho Club. Su población es aproximadamente de 31339 habitantes, todas del área urbana (ONEI, 2015). Las figuras 1 y 2 muestran la ubicación geográfica de la provincia y municipio Santiago de Cuba y de la región estudiada, Distrito José Martí Norte, respectivamente. 


\section{AMAZUNANAS}

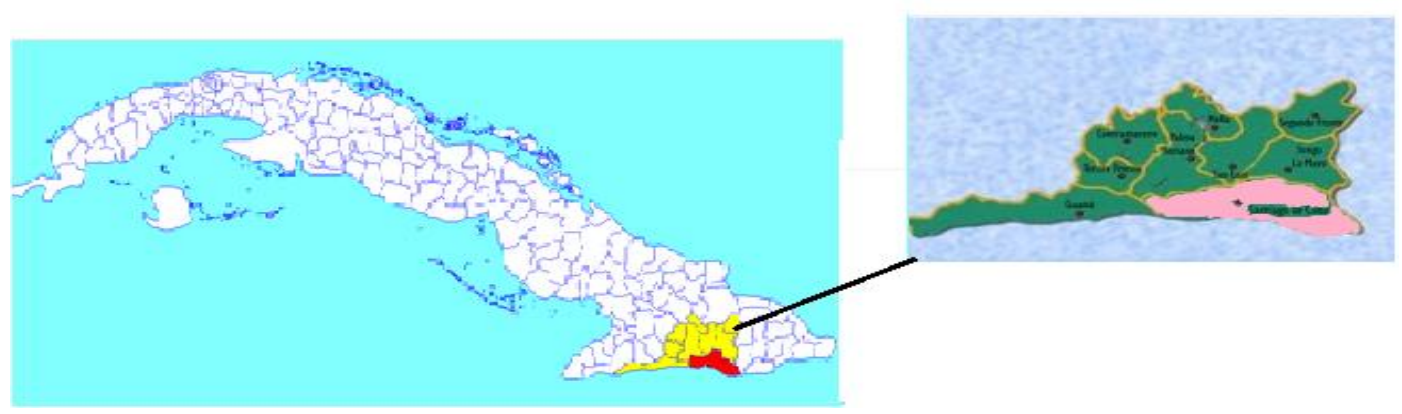

Figura 1. Ubicación geográfica de la provincia y municipio Santiago de Cuba.

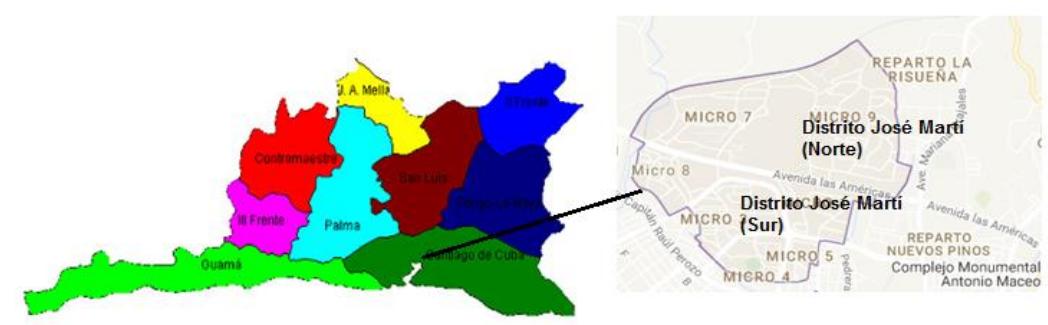

Figura 2. Ubicación geográfica del Distrito José Martí Norte, municipio Santiago de Cuba.

\section{Universo y muestra de estudio}

El universo de estudio estuvo constituido por los pobladores del Consejo Popular "Distrito José Martí Norte" del municipio Santiago de Cuba. Se utilizó una muestra heterogénea, constituida por personas de ambos sexos y en edades superiores a los 20 años, incluyendo yerberos, amas de casas, jubilados y pobladores en general que vivan, estudien o trabajen en el área objeto de estudio, teniendo en cuenta siempre, el consentimiento de participación de los informantes para cooperar con la investigación.

\section{Recolección e identificación taxonómica de la especie vegetal}

Se recolectó el material vegetal cumpliendo con las técnicas de herborización para muestras botánicas, y luego se procedió a la identificación taxonómica en el Centro Oriental de Ecosistemas y Biodiversidad (BIOECO), de la provincia de Santiago de Cuba. Esta fue mostrada a cada informante para comprobar la correspondencia de la especie vegetal en estudio.

\section{Recogida de la información}

La información fue recogida a través de una entrevista apoyada de una encuesta etnobotánica semiestructurada que establece el Programa de Investigación aplicada a la Medicina Popular del Caribe (TRAMIL, 2018) con algunas modificaciones que responde a los objetivos de la investigación. Los datos obtenidos de la encuesta fueron recopilados y organizados en hojas de cálculo de Excel del paquete Microsoft Windows 2007, creada con este propósito. Esto permitió la caracterización de la muestra de estudio atendiendo a la distribución del número total de informantes por edad, sexo, nivel de escolaridad, ocupación, conocimiento de la especie en estudio, usos de la misma, parte de la planta empleada y forma de utilización, método de preparación, vía de administración y usos medicinales más frecuentes. 


\section{AMAZONAS}

\section{Análisis cuantitativo}

Las frecuencias de las citaciones asociadas al conocimiento tradicional de la planta medicinal fueron utilizadas para el análisis cuantitativo. Se determinaron los valores de los índices etnobotánicos como: índice de valor de uso y de nivel de uso significativo a partir de las fórmulas mostradas a continuación.

Índice de valor de uso: Este valor expresa la importancia o valor cultural de una especie determinada para todos los informantes entrevistados. Para el cálculo del índice de valor de uso (IVU) de la especie se empleó la metodología propuesta por Phillips en 1996.

\section{$I V U=\Sigma U v i s / N i s$}

Dónde: Uvis es el número de usos mencionados por cada informante para la especie Nis es el número de informantes entrevistados.

Nivel de uso significativo: Esta metodología expresa que aquellos usos medicinales que son citados con una frecuencia superior o igual al 20\%, por las personas encuestadas que usan plantas como primer recurso para un determinado problema de salud, pueden considerarse significativos desde el punto de vista de su aceptación cultural y, por lo tanto, merecen su evaluación y validación científica (Pérez et al., 2011). Para estimar el Nivel de uso significativo (NUS) para la especie y verificar su aceptación cultural se utilizó la metodología propuesta por Germosén-Robineau en 1995.

\section{NUS=Uso especie $x$ 100/Nis}

Dónde: Uso especie es el número de citaciones para la especie.

\section{Resultados}

\section{Características de la muestra de estudio}

Se entrevistaron un total de 500 pobladores, pertenecientes a las diferentes localidades que conforman el área de estudio, de ellos 320 corresponden al sexo femenino (64\%) y 180 al masculino (36\%). Los informantes fueron distribuidos en seis grupos etarios (1) 20-30, (2) 3140 , (3) 41-50, (4) $51-60$, (5) 61-70 y (6) >70 años, encontrándose predominio en el grupo comprendido entre los 31-40 años con 140 informantes. Del total de los entrevistados, 184(36,8\%) tienen nivel Bachiller y 343(68,6\%) son trabajadores.

\section{Comportamiento del conocimiento y uso de la especie Lawsonia inermis L}

De las 500 personas entrevistadas, 426 de ellas tienen conocimiento de la especie vegetal, representando el $85,2 \%$. Al analizar a este grupo de conocedores respecto al uso o no de la planta, se observó que de las 426 personas que conocían la misma, 327 (76,7\%) de ellas la utilizaban con fines curativos.

\section{Usos reportados}

Se reportó como único uso de la Lawsonia inermis L el medicinal. En el gráfico 1 se muestran los usos medicinales reportados por los informantes, siendo el más notorio el de calmar los nervios con 312 citaciones, representando el 95,4\%. Se reportaron además otros usos, con un menor número de citaciones, como el de bajar la presión arterial y los empleados para tratar el sarpullido, el catarro, la diabetes, el reuma y el dolor de cabeza. 


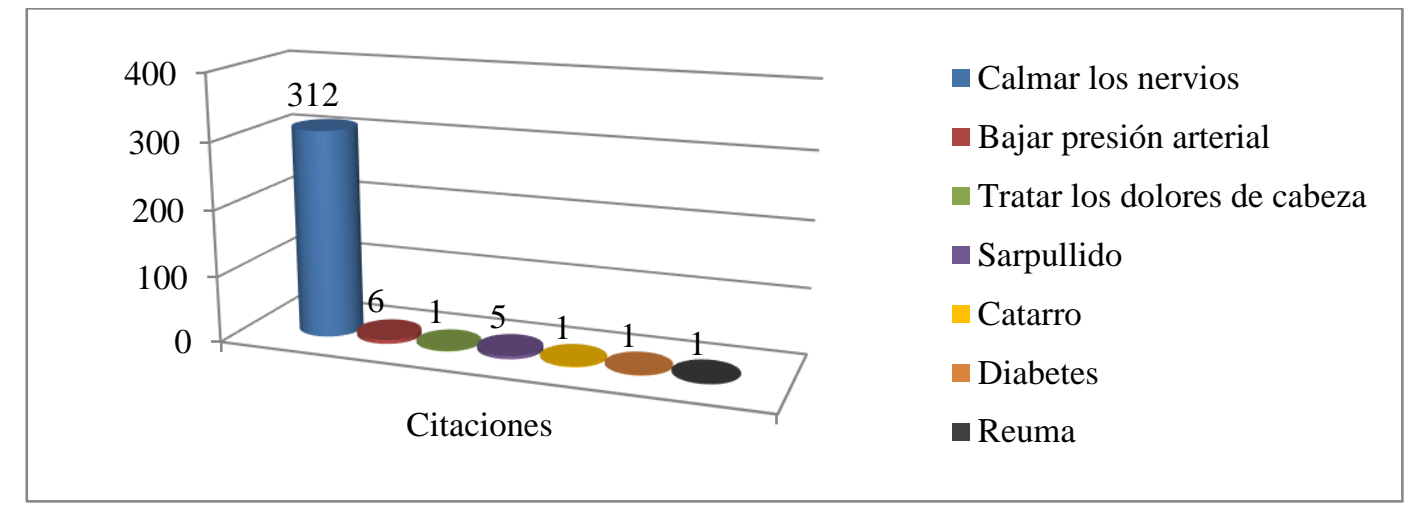

Gráfico 1. Usos reportados para la especie vegetal.

Partes de la planta, forma de utilización, preparación y vías de administración más empleadas

En el gráfico 2 se representa las partes de la planta utilizadas por la población que conforma la muestra de estudio. Nótese que la parte de la planta más utilizada corresponde a las partes aéreas (ramas) con un $41 \%$, seguida en orden decreciente de las hojas (31\%) y las flores (26\%) y, por último, con solo el $2 \%$, los frutos.

La forma de utilización de la planta más referida fue en estado fresco representando el 65 $\%$, según se muestra en el gráfico 3, sin embargo, un $35 \%$ la usa indistintamente tanto en estado fresco y seco. Ninguno de los informantes reportó la utilización de la especie en estado seco.

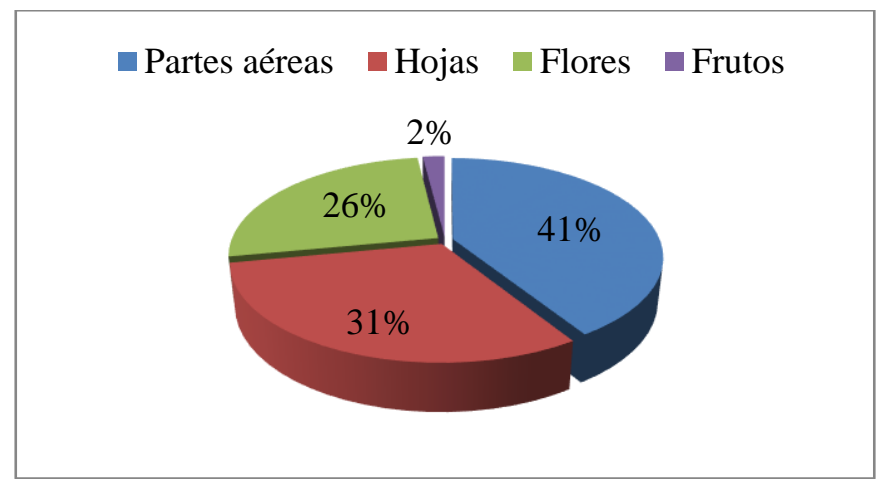

Gráfico 2. Partes de la especie más utilizadas.

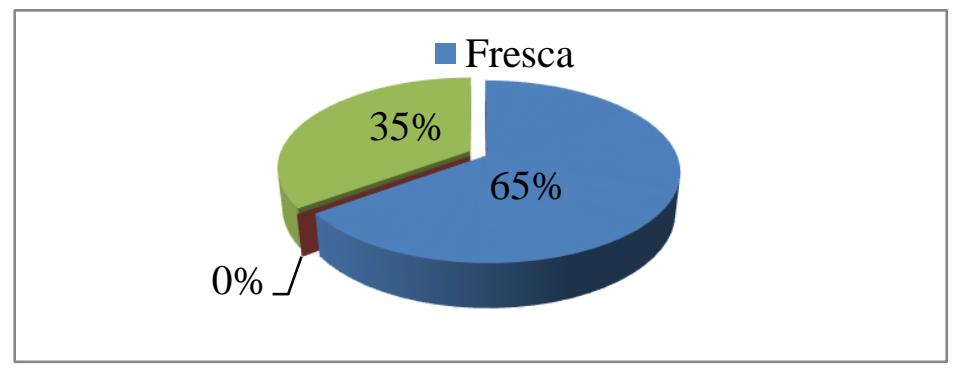

Gráfico 3. Forma de utilización de la planta medicinal 


\section{AMAZÓNAS}

Las preparaciones medicinales reportadas fueron la infusión, la decocción, y una pequeña parte de la muestra informó el uso de ambos métodos. En el gráfico 4 se muestra el comportamiento del uso de estas preparaciones, observándose que el $91 \%$ de los informantes utilizaban el método de infusión para la obtención del preparado medicinal.

En el gráfico 5 se representa el comportamiento de las formas de administración de la especie por la muestra estudiada. El mayor porciento lo tiene la vía de administración oral representada en un $98 \%$, y en menor cuantía la vía tópica (2\%).

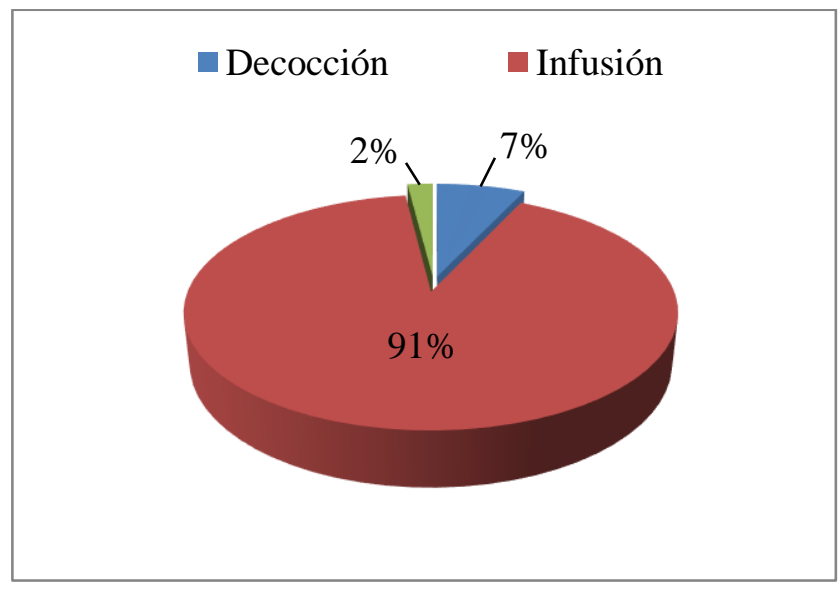

Gráfico 4. Preparaciones medicinales empleadas.

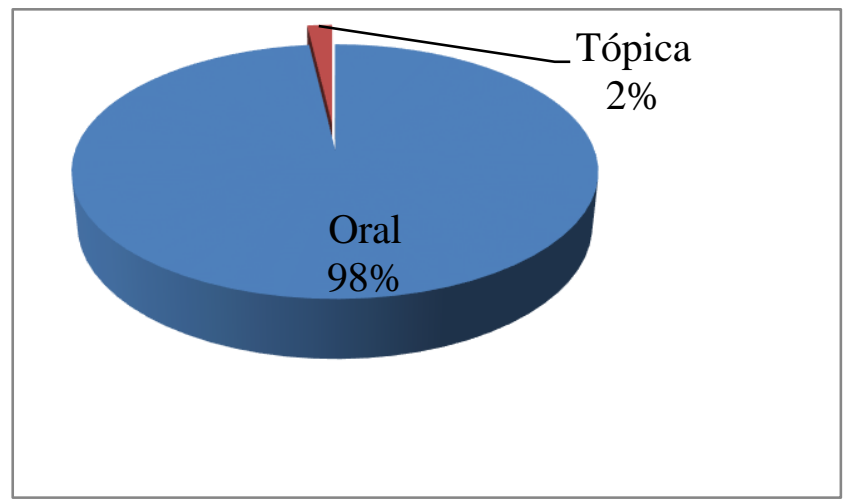

Gráfico 5. Distribución de las formas de administración de la especie.

\section{Indicadores cuantitativos del estudio realizado}

\section{Índice de valor de uso (IVU) y nivel de uso significativo (NUS)}

Los índices de uso calculados para la especie Lawsonia inermis L, correspondientes al Consejo Popular "Distrito José Martí Norte" se muestran en la tabla 1. Los resultados arrojaron un valor de IVU igual a 0.65 y un NUS del $65 \%$. 


\section{AMAZUNANAS}

Tabla 1.

Índice de valor de uso (IVU) y nivel de uso significativo (NUS) de la especie en el Consejo Popular.

\begin{tabular}{|r|r|r|r|}
\hline Comunidad & $\begin{array}{r}\text { No. } \\
\text { Citaciones }\end{array}$ & IVU & $\begin{array}{r}\text { NUS } \\
(\%)\end{array}$ \\
\hline Consejo Popular “Distrito José Martí Norte” & 327 & 0,65 & 65 \\
\hline
\end{tabular}

$\mathrm{Al}$ realizar este análisis por las localidades que conforman la región objeto de estudio, mostrados en la tabla 2, observamos que el mayor número de citaciones para la especie vegetal se correspondió a la localidad del micro 9 con un IVU de 0,94 y un NUS del $94 \%$. Resultados similares se obtuvieron para las localidades Los Cocos con un IVU igual a 0,90 y NUS de 90\%; las localidades de Altos de Quintero y Bajo Rancho Club obtuvieron los mismos valores de IVU igual a 0,89 y un NUS de $89 \%$.

Tabla 2.

Índice de valor de uso (IVU) y nivel de uso significativo (NUS) de la especie por localidades.

\begin{tabular}{|c|c|c|c|}
\hline Localidades & No. Citaciones & IVU & NUS \\
\hline Micro 9 & 92 & 0,94 & 94 \\
\hline Los Cocos & 87 & 0,90 & 90 \\
\hline Micro 10 & 76 & 0,84 & 84 \\
\hline La Risueña & 49 & 0,64 & 64 \\
\hline Altos de Quintero & 67 & 0,89 & 89 \\
\hline Bajo Rancho Club & 58 & 0,89 & 89 \\
\hline
\end{tabular}

\section{Discusión}

El análisis del comportamiento del conocimiento y uso de la Lawsonia inermis L. revela que la especie es conocida por la mayoría de los pobladores del área estudiada, lo que indica que en esta zona existe una rica información y/o cultura acerca del manejo de las plantas medicinales formando parte de la práctica cotidiana como primera vía alternativa en el tratamiento de los problemas de salud. Este nuevo estadío cultural pudo estar asociado a las condiciones económicas excepcionales en la que se encontraba el país a inicios de los años 90 del siglo pasado que conllevó al auge de la medicina alternativa, en la cual el estudio, el cultivo y procesamiento de las plantas con fines terapéuticos ocuparon una posición cimera (Soto, 2002).

El estudio del grupo de conocedores, respecto al uso o no de la planta, mostró que las personas que conocían la misma la utilizaban exclusivamente con fines medicinales, con el objetivo de aprovechar las diversas propiedades farmacológicas que le son atribuidas a la especie vegetal para el alivio de diversos síntomas y enfermedades, lo cual coincide con estudios realizados para la Lawsonia inermis L. donde informan el amplio uso popular y tradicional de la especie (Gagandeep et al., 2010; Kamal, Jawaid, 2010; Santosh et al., 2013; Mamoona et al., 2016).

Como se puede constatar, el uso reportado para la especie con mayor prevalencia, en la muestra de estudio, resultó ser el de calmar los nervios, lo cual pudiera estar asociado al hecho de que en la actualidad las circunstancias personales, laborales, y sociales, generan grandes niveles de tensión y estrés, que producen en el individuo un estado de inquietud y malestar, que en muchos casos, desencadena graves dolencias físicas y a su vez trastornos nerviosos como son la ansiedad, el insomnio, la irritabilidad y la depresión (Del Hoyo, 2004), por lo que la población recurre a las 


\section{AMAZÉNAS}

plantas medicinales como recurso alternativo para tratar sus dolencias, siendo la resedá una de las especies vegetales empleada para el tratamiento de las afecciones nerviosas por su efecto sedante reportado (Sharma et al., 2016); resultado este que coincide con el estudio realizado en Cuba, por Hernández J. en el 2004. Lo que pudiera estar asociado a la actividad farmacológica que exhiben los metabolitos secundarios presentes en la composición química de la especie. Según reportes, se ha identificado la presencia de aceites esenciales y cumarinas, metabolitos estos responsables de la acción hipnótica y sedante (Gagandeep et al., 2010; Santosh et al., 2013; Mamoona et al., 2016; Sharma et al., 2016).

El resto de los usos medicinales de la planta citados en menor cuantía en el estudio, se encuentran reportados en las bibliografías especializadas (Gagandeep et al., 2010; Jain et al., 2010; Santosh et al., 2013; Chaibi et al., 2015; Sharma et al., 2016), no saliendo a relucir nuevas aplicaciones o usos que se le pudiera conferir a algunas de las partes o a la planta en general, con fines curativos.

Resulta común que las partes aéreas (ramas) de la Lawsonia inermis L. sean la parte de la planta más utilizada por los pobladores del área estudiada, ya que si se tiene en cuenta sus características fisiológicas de ser un árbol pequeño, lampiño, muy ramificado, con hojas y flores relativamente pequeñas (Roig, 2012), al recolector le resulta más conveniente tomar las partes aéreas o ramas finas de la planta para la obtención del producto medicinal, encontrándose en esta una gran variedad de metabolitos secundarios que tienen numerosas propiedades farmacológicas útiles en el tratamiento de las enfermedades (El Babili et al., 2013). Estudios llevados a cabo para la especie Lawsonia inermis L. (Guha et al., 2011; Chaibi et al., 2015; Kumar et al., 2017) han demostrado que todas las partes de la planta tienen propiedades farmacológicas, atribuibles a la composición química que presentan, de ahí que también puedan ser usadas también las flores y las hojas, siendo esta última la más utilizada con frecuencia, por ser el órgano de la planta más abundante, asequible y cuyo uso permite el adecuado manejo y preservación de las especies vegetales (Miranda, Cuéllar, 2001).

Es válido destacar, que las personas consumidoras de remedios caseros a partir de plantas medicinales, obtenidas generalmente de los cultivos de los patios, jardines o fincas, acostumbran primero a recolectar el material vegetal, luego al lavado y finalmente a su preparación por métodos tradicionales, sin aplicar previamente técnicas de mejoramiento de la droga como la molienda, humectación o secado, empleados para la producción local y dispensarial de medicamentos herbarios (Claus, Tyler, 1989), por lo que la población usa comúnmente las plantas en su condición natural que es el estado fresco, coincidiendo esto con otras bibliografías consultadas (Martínez et al., 2000; Rodríguez et al., 2007). Se plantea, además, que es en este estado donde la planta posee la mayor cantidad de metabolitos activos responsables de la acción farmacológica (Miranda, Cuéllar, 2001).

El método de infusión resultó el modo de preparación más empleado, por la muestra estudiada, para la obtención del producto medicinal. Este método fácil y sencillo denominado también apagado o té, forma parte de la cultura de consumo de las hierbas aromáticas (Miranda, Cuéllar, 2001) En disímiles investigaciones de consumo de plantas medicinales reportan este método, además del método de decocción, como una de las formas más comunes de preparación de las plantas o fórmulas populares por la población. (Cakilcioglu, Turkoglu, 2010; Clement et al., 2015; Bulut et al., 2017; Heredia et al., 2018).

Según el reporte dado por los entrevistados sobre la forma de administración de la planta, la vía oral resultó ser la más empleada, por ser sin duda, la más fisiológica utilizada para la administración natural de alimentos y medicamentos, debido a que presenta indudables ventajas por su sencillez, seguridad y comodidad (Vila, 2005). 
Respecto al análisis cuantitativo realizado para la especie Lawsonia inermis L., teniendo en cuenta los indicadores etnobotánicos calculados (IVU y NUS), podemos inferir que la misma es ampliamente utilizada con fines medicinales, por los pobladores del Consejo Popular "Distrito José Martí Norte", sobresaliendo la localidad Micro 9 con un mayor uso significativo de la especie, que puede ser el resultado del conocimiento empírico arraigado a la cultura de la población de esta localidad. Los resultados muestran la importancia que tiene esta especie en el tratamiento de las enfermedades, principalmente en las afecciones nerviosas como la ansiedad y el insomnio, y a su vez, evidencian la evaluación y validación farmacológica dirigida a la búsqueda de principios activos y nuevas alternativas terapéuticas que permitan el uso racional, seguro y eficaz de la misma.

\section{Conclusiones}

Se informaron un total de siete usos para la especie Lawsonia inermis L., siendo el más reportado el de calmar los nervios. Las partes aéreas de la planta fue la más empleada, predominando la infusión como forma de preparación administrada por vía oral. El índice de valor de uso obtenido para el Consejo Popular "Distrito José Martí Norte" fue de 0.65 y un $65 \%$ de nivel de uso significativo, siendo la localidad del Micro 9 la que mostró tener un mayor conocimiento del uso de la planta.

\section{Referencias bibliográficas}

Beyra, A., León, M.C., Iglesias, E., Ferrándiz, D., Herrera, R., Volpato, G., Godínez, D., Guimarais, M., y Âlvarez, R. (2004). Estudios etnobotánicos sobre plantas medicinales en la provincia de Camagüey (Cuba). Anal Jard Bot Madrid. 61(2), 185-204.

Blanché, C., Bonet, M.A., Muntané, J. y Vallès, J. (1996). Bases de datos en etnobotánica: elaboración de los resultados. Monografías del Jardín Botánico de Córdoba. 3, 63-68.

Bulut, G., Bozkurt, M.Z., y Tuxlac, E. (2017). The preliminary ethnobotanical study of medicinal plants in Uşak (Turkey). Marmara Pharm J. 21(2), 1-6

Cakilcioglu, U., y Turkoglu, I. (2010). An ethnobotanical survey of medicinal plants in Sivrice (Elazı g-Turkey). Journal of Ethnopharmacology. 132, 165-175.

Chaibi, R., Romdhane, M., Ferchichi, A., y Bouajila, J. (2015). Assessment of antioxidant, antiinflammatory, anti-cholinesterase and cytotoxic activities of Henna (Lawsonia inermis) flowers. Journal of Natural Products. 8, 85-92.

Claus, P., y Tyler, E. (1989). Farmacognosia. La Habana, Cuba: Ed. Revolucionaria. pp. 8-9

Clement, N., Baksh-Comeau, S., y Seaforth, E. (2015). An ethnobotanical survey of medicinal plants in Trinidad. Journal of Ethnobiology and Ethnomedicine. 11:67, pp. 1-28.

Del Hoyo, M.A. (2004). Estrés laboral. Documento divulgativo. Ed. Instituto Nacional de Seguridad e Higiene en el Trabajo. Madrid: ISBN: 84-7425-474-4. Disponible en: https://www.sesst.org/2Fwp-content/2Fuploads/2F2018/2F08/2Festres-laboral-guia.pdf. [Acceso: 30 may. 2018].

El Babili, F., Valentin, A, y Chatelain, Ch. (2013). Lawsonia inermis: Its Anatomy and its Antimalarial, Antioxidant and Human Breast Cancer Cells MCF7 Activities. Pharmaceut Anal Acta. 4(1), 2-6

Gagandeep, C., Sandeep, G., y Priyanka, P. (2010). Lawsonia inermis Linnaeus: A Phytopharmacological Review. International Journal of Pharmaceutical Sciences and Drug Research. 2(2), 91-98

Germosén-Robineau, L. (1995). Hacia una farmacopea vegetal caribeña. Edición TRAMIL 7. Santo Domingo: Enda Caribe, UAG \& Universidad de Antioquia.

Guha, G., Rajkumar, V., Kumar, R., y Mathew, L. (2011). Antioxidant activity of Lawsonia inermis extracts inhibits Chromium (VI)-Induced cellular and DNA toxicity. EvidenceBased Complementary and Alternative Medicine. Vol. 2011, 1- 9 


\section{AMAZONAS}

Heredia, Y., García, J., López, T., Chil, I., Arias, D., Escalona, J.C., González, R., Costa, J., Suarez, D., Sánchez, M., y Martínez, Y. (2018). Estudio etnobotánico de las plantas medicinales usadas por los habitantes de Holguín, Región Oriental, Cuba. Boletín Latinoamericano y del Caribe de Plantas Medicinales y Aromáticas. 17(2), 160 - 196

Hernández, J., y Volpato, G. (2004). Herbal mixtures in the traditional medicine of Eastern Cuba. Journal of Ethnopharmacology. 90, 293-316 (pendiente agregar en el texto a Volpato)

Illnait, JF. (2007). Principales referencias etnomédicas sobre el Anamú. Revista CENIC. Ciencias biológicas. 38(1), 27-30.

Jain, C., Shah, P., Sonani, G., Dhakara, S., y Patel, M. (2010). Pharmacognostical and Preliminary Phytochemical Investigation of Lawsonia Inermis L. Leaf. Rom. J. Biol. - Plant Biol. 55(2), 127-133

Kamal, M, y Jawaid, T. (2010). Pharmacological activities of Lawsonia inermis Linn: a review. IJBR. 1(2), 62-68.

Kumar, M., Chandel, M., Kaur, P., Pandit, K., Kaur, V., y Kaur, S. (2016). Chemical composition and inhibitory effects of water extract of henna leaves on reactive oxygen species, DNA scission and proliferation of cancer cells. EXCLI Journal. 15, 842-857

Kumar, M., Kaur, P., Chandel, M., Singh, A.P., Jain, A., y Kaur, S. (2017). Antioxidant and hepatoprotective potential of Lawsonia inermis L. leaves against 2-acetylaminofluorene induced hepatic damage in male Wistar rats. BMC Complementary and Alternative Medicine. 17:56. 1-11

Macías, E., Coy, D., y Suárez, E. (2011). Análisis fitoquímico preliminar y actividad antioxidante, antiinflamatoria y antiproliferativa del extracto etanólico de corteza de Zanthoxylumfagara (L.) Sarg. (Rutaceae). Revista Cubana de Plantas Medicinales. 16(1), 43-53.

Mamoona, N., Muhammad, H.Z., Abdul, G. (2016). Complete Prospective of Lawsonia inermis Linn- Review. Imperial Journal of Interdisciplinary Research. 2(2), 190-197.

Martin, G.J. (1995). Ethnobotany: a methods manual. Londres: Chapman \& Hall.

Martínez, J.V., Bernal, Y., Henry, A., y Cáceres, A. (2000). Fundamentos de agrotecnología de cultivo de plantas medicinales iberoamericanas. Revista Cubana de Plantas Medicinales. 5(3), 125.

Miranda, M, y Cuéllar, A. (2001). Farmacognosia y Productos Naturales. La Habana, Cuba: Editorial Félix Varela. pp. 68,159.

Ochoa, A., Gross, C., Menéndez, M., Chacón, N., y Torres, M. (1995). Propiedades antinflamatorias del ungüento de Petiveria alliaceae L. Rev Cub Farm. 30(S), 143.

OMS. (2002). Estrategias de la OMS sobre la Medicina Tradicional 2002-2005. WHO/EDM/TRM/2002.1, Ginebra, Suiza. Disponible en: https://www.google.com/search?source=hp\&ei=J-PrXruYBoa1ggevsKoBQ\&q=OMS.+/282002/29.+Estrategias+de+la+OMS+sobre+la+Medicina+Tradiciona 1

ONEI. (2015). Oficina Nacional de Estadística e Información. Anuario Estadístico Santiago de Cuba 2014. Edición 2015. Disponible en: http://www.one.cu/aed2014/34Santiago/20de/20Cuba/Municipios/06/20Santiago/20de/2 0Cuba.pdf

Pardo, M., y Gómez, E. (2003). Etnobotánica: Aprovechamiento tradicional de plantas y patrimonio cultural. Anales Jard. Bot. 60(1), 171-182.

Pérez, M., Sueiro, M., Boffill, M., Morón, F., Marrero, E., Rodríguez, M., Méndez, R., y González, M. (2011). Estudio etnobotánico de las plantas más utilizadas como diuréticas en la Provincia de Villa Clara, Cuba. Bol Latinoam Caribe Plant Med Aromat. 10(1), 4655 .

Pérez, Y., Vázquez, A., Suárez, F., Rodríguez, E., y Baró, Y. (2009). Plantas antidermatofíticas, utilizadas en comunidades costeras del municipio Guamá, Santiago de cuba. Rev. Etnobiol. 7, 56-62 


\section{AMAZONANAS}

Phillips, O. (1996). Some quantitative methods for analyzing ethnobotanical knowledge. In Alexiades, M.N. (ed.). Selected guidelines for ethnobotanical research: A field manual. New York: The New York Botanical Garden, Bronx.

Pimentel, O. (2002). Estudio Etnobotánico de las plantas medicinales en el Valle de San Andrés. Pinar del Río Cuba. Monografias. Disponible en: https://www.monografias.com/2Ftrabajos71/2Festudio-etnobotanico-plantasmedicinales/2Festudio-etnobotanico-plantas-medicinales2.shtml

Rodríguez, Y., Valdés, M., Hernández, H., y Soria, S. (2019). Guía metodológica para estudio etnobotánico de especies forestales en comunidades amazónicas y afines. Revista Cubana de Ciencias Forestales. 7(1), 98-110

Roig, J.T. (2012). Plantas medicinales, aromáticas o venenosas de Cuba. La Habana, Cuba: Editorial Científico-Técnica. pp. 818-819.

Santosh, Y., Anil, K., Jyotsna, D., y Ashok, K. (2013). Essential Perspectives of Lawsonia inermis. International journal of pharmaceutical and chemical sciences. 2(2), 888-896

Sharma, R.K., Goel, A., Bhatia, A.K. (2016). Lawsonia inermis linn: a plant with cosmetic and medical benefits. Int J Appl Sci Biotechnol. 4(1), 15-20

Soto, O.R. (2002). La domesticación de las plantas medicinales en cuba: importancia y experiencias concretas. Biocenosis. 16 (1-2), 66-70.

TRAMIL. (2018). (Traditional Medicine in the Islands). Requerimientos de encuestas. Programa de investigación aplicada a la medicina popular del Caribe, República Dominicana. Encuestas TRAMIL. Disponible en: http://www.tramil.net/es/content/modeloencuestas

Vargas, B., Pupo, Y., Puertas, A., Mercado, I., y Hernández, W. (2011). Estudio etnobotánico sobre tres especies Arvenses en localidades de la región Oriental de Cuba. Revista Granma Ciencia. 15(3).

Vila, J.L. (2001). Tecnología Farmacéutica. Vol. II. España: Editorial Síntesis. p.55. 